\title{
Resultados funcionales de pacientes tratados con faringolaringectomía y reconstrucción faríngea con colgajo pediculado
}

\author{
Glomaryeth Luque S. ${ }^{1}$, Marcelo Fuentes E. ${ }^{1}$, Marcelo Sánchez P. ${ }^{1}$, \\ Andrea San Pedro S. ${ }^{1}$, Ross Mery Ruiz L. ${ }^{1}$ y Dahiana Pulgar B. ${ }^{1,2}$
}

\section{Functional Outcomes using pedicle flap reconstruction approach after pharyngolaryngectomy in laryngeal carcinoma patients}

Introduction: Total laryngectomy is the treatment of choice for advanced laryngeal cancer and after radiotherapy failure. In patients with pharyngeal invasion, it is associated with total or partial pharyngectomy, wich defect can be repaired with free or pedicle flaps. Aim: To present a brief series of pedicle flap reconstruction approach after pharyngolaryngectomy in laryngeal carcinoma patients and functional outcomes Materials and Method: We looked at laryngeal cancer patient records who were treated with total laryngectomy with partial pharyngectomy and pharyngeal reconstruction with pedicle flap at "Hospital Regional de Talca" between the years 2009 and 2017, finding 6 cases, 4 of which were alive at the beginning of the study. We analized videoflourocopy swallow studies to assess mechanisms of deglutition, and presence of stenosis or fistulas. We also performed a nutritional state assessment and a quality of life questionnaire. Results: During videodeglutition study, the 4 patients showed minimal contrast swallow delay at hypopharynx and cervical esophagus. All the patients were found eutrophic in their nutritional assessment and with no significant evidence of quality of life disturbances secondary to deglutition state. Conclusions: Partial pharyngeal reconstruction using pedicle flaps in patients who underwent total laryngectomy with partial pharyngectomy allows to maintain an adequate deglutition without dysphagia, as well as a good nutritional state.

Key word: laringeal cancer; deglutition; videoflouroscopy; pharyngolariyngectomy.

\section{Resumen}

Introducción: La laringectomía total es el tratamiento de elección del cáncer de laringe avanzado, requiriendo en ocasiones asociar una resección parcial o total de la faringe para su manejo. El defecto faríngeo, puede repararse con colgajos libres o pediculados, teniendo estos últimos la ventaja de ser simples, confiables y resistentes a la radioterapia. Objetivo: Presentar los resultados funcionales de una serie de pacientes tratados con faringolaringectomía y reconstrucción faríngea con colgajo pediculado. Material y Método: Se revisaron registros de pacientes con laringectomía total más faringectomía parcial y reconstrucción con colgajo pediculado en el Hospital Regional de Talca entre 2009 y 2017, encontrando 6 casos de los cuales 4 se encontraron vivos al momento de iniciar el estudio. Se realizó videofluoroscopía para evaluar deglución, presencia de estenosis y/o fístulas, además, de evaluación nutricional y encuesta de calidad de vida. Resultados: En el estudio de la deglución por fluoroscopía, todos los pacientes presentaron escasa retención del material de contraste en la hipofaringe y esófago cervical, lo cual está en relación con cambios morfológicos posquirúrgicos, sin afectar significativamente el mecanismo deglutorio. Todos los pacientes se encontraron eutróficos en su evaluación nutricional y sin evidencias de alteración de su calidad de vida secundaria a la deglución. Conclusiones: La reconstrucción faríngea parcial con colgajo pediculado en pacientes con laringectomías totales asociadas a faringectomía parcial permite una deglución adecuada y sin disfagia, con un estado nutricional eutrófico.

Palabras clave: cáncer de laringe; deglución; faringeolaringectomía; colgajo pediculado.
${ }^{1}$ Hospital Regional de Talca. ¿Universidad Católica del Maule.

Recibido el 14 de junio de 2018 y aceptado para publicación el 26 de julio de 2018.

Correspondencia a: Dra. Dahiana Pulgar B. dapulgar@gmail.com 


\section{Introducción}

El cáncer de laringe es una patología oncológica de relevancia mundial, estimándose para 2017 el diagnóstico de 13.360 nuevos casos solo en Estados Unidos ${ }^{1}$. En Chile se estima una incidencia ajustada por edad de 2,12 por cada 100.000 habitantes, y ajustada por sexo de 0,77 por cada 100.000 habitantes para mujeres y de 4,99 por cada 100.000 habitantes para hombres ${ }^{2}$.

Los factores de riesgo del cáncer de laringe, se han identificado claramente, siendo los más importantes el consumo de alcohol, el tabaquismo y la infección por virus papiloma humano (HPV), viéndose con este último un fenotipo clínico diferente y con mejor pronóstico, de predominio en países desarrollados ${ }^{3}$. En nuestro país persiste el predominio epidemiológico del cáncer de laringe HPV-negativo, describiéndose un riesgo relativo de 8,07 para fumadores activos ${ }^{2}$. Estos pacientes son típicamente hombres caucásicos, mayores de 60 años, de nivel socioeconómico medio-bajo, con historia importante de consumo de alcohol y tabaquismo, con tamaño tumoral $(\mathrm{T})$ avanzado $\mathrm{y}$ compromiso linfático $(\mathrm{N})$ precoz al momento del diagnóstico ${ }^{3}$.

El tratamiento del cáncer de laringe, tradicionalmente se ha basado en la resolución quirúrgica mediante laringectomía (total o parcial) o faringolaringectomía. Sin embargo, en las últimas décadas, ha adquirido mayor protagonismo el manejo conservador no quirúrgico (quimioterapia y radioterapia en distintas combinaciones), el cual es utilizado en estadios precoces. En pacientes con enfermedad avanzada (T3, T4) o con mala respuesta a tratamientos previos, la cirugía sigue siendo el manejo de elección ${ }^{3,4}$. Por las características epidemiológicas de la región del Maule con un alto porcentaje de ruralidad, nuestros pacientes son diagnosticados en etapas avanzadas de su enfermedad, requiriendo de extensas resecciones para lograr un adecuado control oncológico, lo que genera importantes defectos a nivel faríngeo.

No existe, actualmente, consenso sobre el método óptimo de reconstrucción faríngea, pudiendo ésta ser realizada con colgajos libres o pediculados ${ }^{5,6}$. El objetivo primario de nuestro estudio fue evaluar los resultados funcionales de los pacientes con cáncer de laringe avanzado tratados con faringolaringectomía y reconstrucción faríngea con colgajo pediculado. Los objetivos específicos fueron evaluar deglución mediante videofluoroscopía, evaluación del estado nutricional actual y determinar el impacto del tratamiento en la calidad de vida.

\section{Material y Método}

\section{Diseño}

Estudio descriptivo. Reporte de casos.

\section{Pacientes}

Se incluyó a todos los pacientes con diagnóstico de cáncer de laringe que fueron tratados con laringectomía total más faringectomía parcial y reconstrucción con colgajo pediculado en el Hospital Regional de Talca entre los años 2009 y 2017.

\section{Método}

Los datos se recolectaron de los registros de fichas clínicas y registros de biopsias de nuestro centro. Para el seguimiento y evaluación de resultados funcionales, los pacientes fueron citados y controlados por equipo multidisciplinario. Se realizó el estudio de los mecanismos de la deglución con visualización fluoroscópica y adquisición de imágenes en modalidad de video (15 a 30 cuadros por segundo), en el seriógrafo marca Siemens, modelo Luminos DRF full con su estación de trabajo, empleándose la administración por vía oral de medio de contraste baritado y/o yodado hidrosoluble, en consistencia líquida, con cantidades progresivas, obteniéndose proyecciones anteroposterior y lateral; además de una evaluación del estado nutricional mediante registro de antropometría y exámenes nutricionales plasmáticos y se evaluó el impacto biopsicosocial del tratamiento mediante la aplicación del cuestionario de la Universidad de Washington sobre calidad de vida en pacientes con cáncer de cabeza y cuello (UW-QOL v4 en español) estandarizado en español ${ }^{7,8}$, el cual evalúa 12 dominios en base a preguntas, teniendo estas entre 3 y 6 alternativas, con puntaje de 0 (peor) a 100 (mejor) de acuerdo a la jerarquía de respuesta ${ }^{7}$. Estos dominios se pueden analizar independientemente o agrupar en 2 subescalas, de función física (masticación, deglución, habla, gusto, saliva y apariencia) y de función socioemocional (ansiedad, ánimo, dolor, actividad, recreación $\mathrm{y}$ función de hombro) $)^{7}$.

\section{Variables}

Las variables consideradas fueron: sexo, edad, factores de riego, comorbilidades, estadio al diagnóstico, cirugía realizada, morbilidad posoperatorio, de alimentación enteral, evaluación fonaudiológica y nutricional, tipo de alimentación actual, hallazgos a la videofluoroscopía de deglución, evaluación antropométrica (peso, talla e índice de masa corporal), niveles de albúmina, prealbúmina, proteínas totales, BUN, creatinina, electrolitos plasmáticos, calcio, 
fósforo, magnesio, perfil de fierro, hemograma y proteína $\mathrm{C}$ reactiva (PCR) y promedios de función física y socioemocional del UW-QOL v4.

\section{Análisis de resultados}

Se utilizó estadística descriptiva con el programa SPSS. 22 (SPSS Inc., Chicago, IL). Para la descripción de variables continuas se utilizó mediana y rango. La descripción de variables cualitativas se realizó mediante frecuencias absolutas y porcentajes.

\section{Resultados}

En el período descrito, 6 pacientes con diagnóstico de cáncer de laringe fueron tratados con laringectomía total más faringectomía parcial y reconstrucción faríngea con colgajo pediculado, de los cuales 4 se encontraron vivos al momento de iniciar el estudio, siendo factible la evaluación de resultados funcionales. La causa de muerte de los 2 pacientes fallecidos, según se consigna en el registro civil, fue progresión de enfermedad en un caso y un evento cardiovascular en el otro.

La totalidad de los pacientes analizados presentaron enfermedad avanzada. El promedio de edad de los pacientes al momento de la cirugía fue de $66 \pm$ 13 años. La Tabla 1 resume las principales características clínicas de los pacientes. La evaluación de resultados funcionales se realizó con una mediana de seguimiento de 8,17 meses posterior a la reconstrucción faríngea (5-100 meses).

\section{Cirugía y hospitalización}

En 3 pacientes la reconstrucción faríngea se realizó en el mismo tiempo quirúrgico con colgajo miocutáneo de pectoral mayor. En un paciente se realizó rafia faríngea primaria, requiriendo reparación con colgajo pediculado en un segundo tiempo operatorio. La estadía en la Unidad de Paciente Crítico presentó una mediana de 9,5 días (6-84 días). La mediana de estadía hospitalaria fue de 42 días (27-93 días). El régimen por vía oral se reestableció en una mediana de 17,5 días (13-90 días).

\section{Estudios realizados}

Estudio de la deglución por videofluroscopía: El contraste utilizado por vía oral fue sulfato de bario al 98\% (E-Z-HD) en 2 pacientes, y contraste yodado hidrosoluble iothalamate meglumine $60 \%$ (Conray) en 2 de ellos. En todos los pacientes se objetivó ausencia de alteraciones en la fase de la deglución oral, con buen pasaje del bolo de contraste hacia la orofaringe, con adecuada elevación del paladar blando y sin reflujo nasofaríngeo. Además, todos los pacientes presentaron escasa tendencia a la retención del material de contraste en la hipofaringe y esófago cervical, lo cual está en relación con cambios morfológicos posquirúrgicos, sin afectar significativamente el mecanismo deglutorio, y sin evidencia de paso del medio de contraste a vía aérea en ninguno de ellos. En un paciente se pesquisó un área de disminución de calibre a nivel de esófago cervical. Además, en otro paciente se objetivó fístula esófagocutánea cervical derecha conocida, de bajo débito (Figura 1).

Estado nutricional: Todos los pacientes al momento de la evaluación se encontraban ingiriendo régimen común con consistencia máxima de sólidos (carnes). El índice de masa corporal (IMC) promedio fue de $26,1 \pm 2,37$. Tres pacientes $(75 \%)$, presentaron incremento de peso en relación con el presentado preoperatorio. Dentro de los exámenes de laboratorio, todos los pacientes presentaron glicemia, electrolitos plasmáticos (sodio, potasio, cloro, magnesio y fósforo), proteínas totales, nivel de leucocitos y función renal en rangos normales. Dentro de las alteraciones encontradas, un paciente presentó hipoalbuminemia de $3,3 \mathrm{~g} / \mathrm{dl}$, otro paciente presentó calcemia de $7,53 \mathrm{mg} / \mathrm{dl}, 2$ presentaron discreta elevación de PCR (35,9 y 66,5 mg/dl) y 3

Tabla 1. Características demográficas y clínicas de los pacientes $(n=4)$

\begin{tabular}{|c|c|}
\hline Variable & $\mathbf{N}^{\circ}$ de pacientes \\
\hline $\begin{array}{l}\text { Sexo } \\
\text { - Femenino } \\
\text { - Masculino }\end{array}$ & $\begin{array}{l}0 \\
4(100 \%)\end{array}$ \\
\hline $\begin{array}{l}\text { Historia de tabaquismo } \\
\text { - Sí } \\
\text { - No }\end{array}$ & $\begin{array}{l}3(75 \%) \\
1(25 \%)\end{array}$ \\
\hline $\begin{array}{l}\text { Clasificación tamaño tumoral }(\mathrm{T}) \\
\text { - } \mathrm{T} 1 / \mathrm{T} 2 \\
\text { - } \mathrm{T} 3 / \mathrm{T} 4\end{array}$ & $\begin{array}{l}1(25 \%) \\
3(75 \%)\end{array}$ \\
\hline $\begin{array}{l}\text { Compromiso nodal }(\mathrm{N}) \\
\text { - } \mathrm{N} 0 / \mathrm{N} 1 \\
\text { - } \mathrm{N} 2 / \mathrm{N} 3\end{array}$ & $\begin{array}{l}2(50 \%) \\
2(50 \%)\end{array}$ \\
\hline $\begin{array}{l}\text { Compromiso a distancia }(\mathrm{M}) \\
\text { - } \mathrm{M} 0 \\
\text { - } \mathrm{M} 1\end{array}$ & $\begin{array}{l}4(100 \%) \\
0\end{array}$ \\
\hline $\begin{array}{l}\text { Recurrencia/persistencia enfermedad } \\
\text { - Sí } \\
\text { - No }\end{array}$ & $\begin{array}{l}2(50 \%) \\
2(50 \%)\end{array}$ \\
\hline $\begin{array}{l}\text { Presencia de comorbilidades } \\
\text { - Sí } \\
\text { - No }\end{array}$ & $\begin{array}{l}4(100 \%) \\
0\end{array}$ \\
\hline
\end{tabular}




\section{ARTÍCULO ORIGINAL}

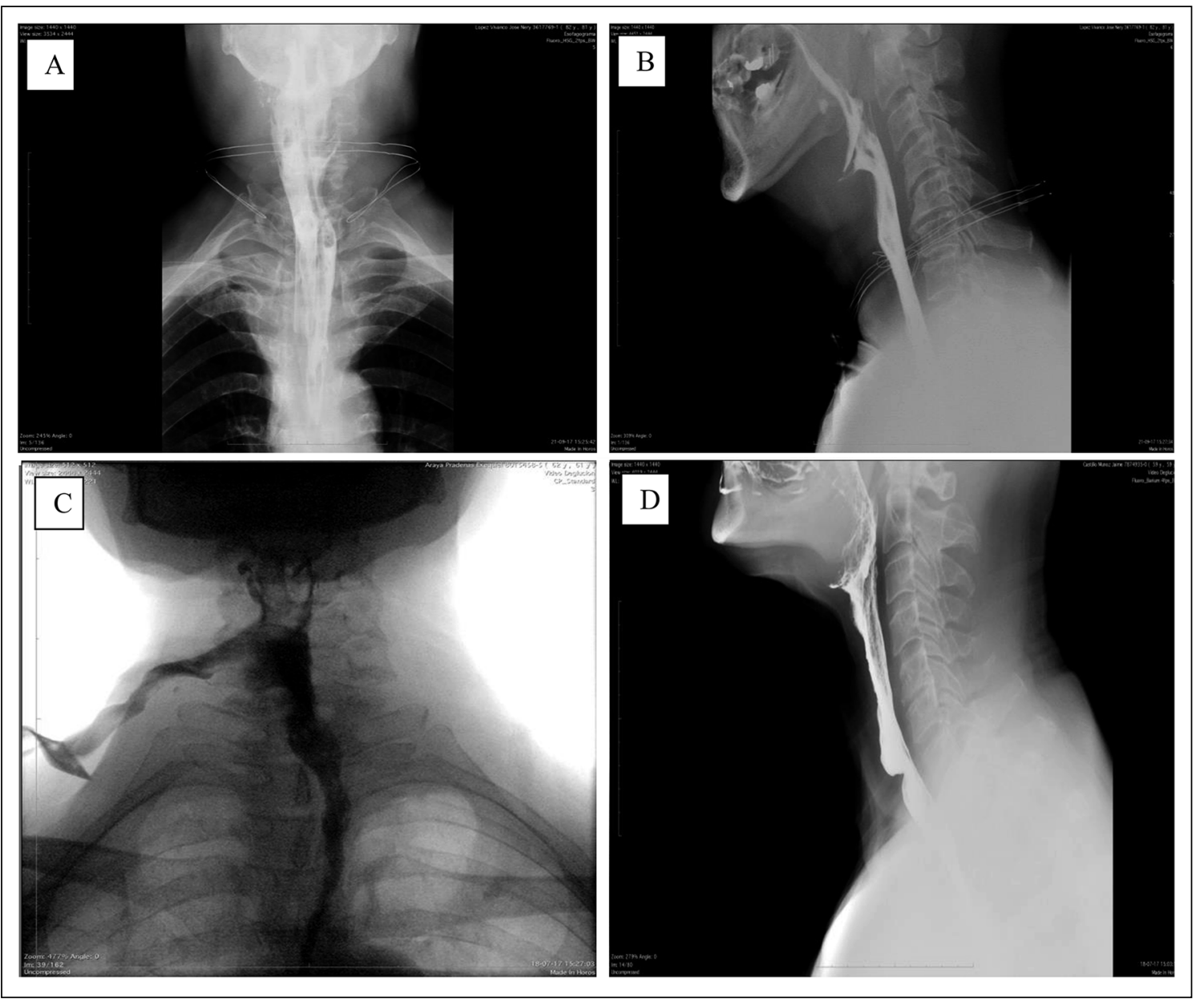

Figura 1. Hallazgos en videodeglución de pacientes con reconstrucción faríngea parcial con colgajo pediculado. Videodeglución posterior a reconstrucción parcial de faringe. A: Proyección AP: Cambios posquirúrgicos esofágicos con adecuada restitución funcional del tránsito. B: Proyeción lateral: Cambios posquirúrgicos. Sin evidencia de estenosis ni filtraciones, conservándose el adecuado transito esofágico. C: Presencia de fístula esofagocutánea cervical derecha. D: Leve estenosis esofágica cervical, apreciada en la proyección lateral, que condiciona escasa retención del material contrastado durante el tránsito esofágico.

evidenciaron anemia, con un hematocrito promedio de $37,2 \pm 3,2 \%$, y una hemoglobina promedio de $11,9 \pm 1,2 \mathrm{mg} / \mathrm{dl}$, solo uno de ellos con un patrón microcítico-hipocrómico (VCM 69,5 fl y CHCM $30,7 \mathrm{~g} / \mathrm{dl}$ ) asociado a hipoferremia de $18,9 \mu \mathrm{g} / \mathrm{dl}$.

Calidad de vida: Tres pacientes requirieron asistencia para completar el cuestionario. Todos los pacientes reportaron un puntaje de 100 en los apartados de deglución y masticación. El puntaje promedio de función física fue de $81,5 \pm 15,3$ puntos, mientras que el puntaje promedio de la función socioemocional fue de 71,3 $\pm 23,26$ puntos, con un puntaje promedio global de $76,35 \pm 17,97$ puntos.

\section{Discusión}

El manejo del cáncer de laringe ha variado en el transcurso del tiempo, tomando más relevancia en las últimas décadas el tratamiento conservador con el uso de radioterapia y quimioterapia ${ }^{3,9}$. Sin embargo, el tratamiento no quirúrgico se asocia a peores resultados oncológicos en pacientes con enfermedad avanzada, para los cuales la laringectomía total sigue mostrando mayores beneficios ${ }^{4,9}$. En el reporte de pacientes de nuestro centro se indicó tratamiento con laringectomía total a pacientes con estadios avanzados $\mathrm{y} / \mathrm{o}$ con mala respuesta a tratamiento conservador de laringe con intención curativa pre- 
vio, lo cual se correlaciona, además, con el tipo de pacientes y sus factores de riesgo para el desarrollo de la enfermedad ${ }^{3,4,10,11}$. Pese a que la infección por HPV podría estar asociada a un incremento en el riesgo de carcinoma escamoso de laringe, guías internacionales establecen que no existe evidencia para recomendar la detección rutinaria de HPV en cáncer de cabeza y cuello de ubicación diferente a orofaringe ${ }^{12}$.

Anatómicamente la laringe se relaciona y comunica directamente con la faringe, compartiendo una pared, por lo que esta última puede terminar con un amplio defecto posterior a la resección de un cáncer de laringe avanzado. La reconstrucción del defecto faríngeo secundario a la laringectomía total puede ser reparado mediante cierre primario o con utilización de colgajos libres o pediculados, en casos donde se requiera de tejido adicional para realizar un cierre sin tensión, minimizar las filtraciones y el desarrollo posterior de estenosis ${ }^{13}$.

Desde su descripción en 1879, el colgajo miocutáneo de pectoral mayor ha sido uno de los más utilizados en la reconstrucción de defectos cervicales ${ }^{14,15}$ debido a poseer abundante tejido, incluyendo musculatura y piel, además de una técnica quirúrgica simple, rápida y en supino ${ }^{14}$. Además, en comparación con otras técnicas reconstructivas, ha demostrado ser el colgajo con menor morbilidad, reflejándose en resultados funcionales como la recuperación de ingesta vía oral en menor tiempo ${ }^{15}$. Sin embargo, actualmente los colgajos libres podrían considerarse de primera elección para la reconstrucción de los defectos faríngeos, debido a su mayor versatilidad, compatibilidad de tejidos, función, resultado estético, y menor morbilidad del sitio donante ${ }^{16}$, relegando a los colgajos pediculados a un rol de salvataje en casos de fracaso del colgajo libre. Lee et al. ${ }^{17}$, en un estudio retrospectivo que incluyó a 99 pacientes con reconstrucción de hipofaringe con colgajo libre $(\mathrm{n}=85)$ o colgajo pediculado de pectoral mayor $(n=14)$, no reportaron superioridad funcional con el uso de colgajos libres, los que a su vez se asociaron con mayor tasa de morbilidad posoperatoria especialmente respiratoria. En nuestro reporte, todos los pacientes fueron reconstruidos mediante el uso de colgajos miocutáneos pediculados. Consideramos que la selección de un método reconstructivo apropiado debe tener en cuenta tanto los factores del paciente (edad, presencia de comorbilidades, fracaso de tratamiento conservador previo) como los factores del cirujano (entrenamiento en técnicas microquirúrgicas) y de la institución (equipamiento).

El estudio de mecanismos de la deglución mediante videofluoroscopía puede identificar altera- ciones de variadas fases de la deglución, teniendo en cuenta además los cambios morfológicos y funcionales secundarios a la intervención quirúrgica. En nuestros pacientes el análisis se centró en la identificación de alteraciones en la velocidad del bolo, la presencia de volumen residual, aspiración, estrechez/estenosis y/o fístulas. Se ha reportado sobreestimación de alteraciones funcionales de la deglución en los hallazgos imagenológicos en comparación con el estado deglutorio referido por los pacientes, lo cual puede explicarse por cambios morfológicos esperables posterior a la cirugía, que impresionen como alteraciones en los mecanismos deglutorios, sin traducirse en una alteración funcional para el paciente ${ }^{18,19}$. Mencionamos el hallazgo de una fístula esofagocutánea de bajo débito en uno de nuestros pacientes, bien tolerada por este, quien adecúa su deglución mediante manipulación del ostium fistular y ha rechazado intervenciones quirúrgicas adicionales para el manejo de ésta. Dicha fístula, se da en el contexto de una cirugía de salvataje sobre territorio previamente irradiado, el cual es ampliamente conocido por poseer mayor tasa de complicaciones posoperatorias, variando entre $37 \%$ y $74 \%$ en diversas series, explicado por los cambios moleculares en la fisiología de la reparación de tejidos ${ }^{15,20-22}$.

El estado nutricional de los pacientes tratados por cáncer de cabeza y cuello es un aspecto fundamental no sólo como reflejo de su estado funcional, sino como parámetro con alto valor predictor de su sobrevida ${ }^{23}$. Los parámetros más confiables y mayormente disponibles para interpretar el estado nutricional de los pacientes son la diferencia de peso (\%), la presencia de anemia, hipoalbuminemia y el alza de parámetros inflamatorios, los cuales se pueden contrastar con una evaluación de ingesta dietaria $^{23,24}$. Los resultados obtenidos en nuestros pacientes reflejan un buen estado nutricional general, con tendencia a la recuperación de peso, lo que se correlaciona con la ausencia de IMC bajo peso, el cual posee valor clínico limitado al ser utilizado como parámetro único ${ }^{23}$. De este análisis se desprende también la importancia de una evaluación nutricional preoperatoria para el óptimo manejo nutricional posoperatorio ${ }^{24}$, el cual fue realizado en nuestros pacientes.

La calidad de vida de los pacientes laringectomizados puede verse afectada por una cantidad considerable de aspectos, y su identificación oportuna entrega herramientas invaluables al tratante para un abordaje integral durante el seguimiento. El cuestionario de la Universidad de Washington de calidad de vida está diseñado específicamente para pacientes 
con cáncer de cabeza y cuello, destacándose por ser una medición simple y a la vez clínicamente relevante, capaz de ser aplicada en los controles de rutina, teniendo como ventajas el ser breve, multifactorial y detallado, permitiendo pesquisar cambios sutiles $^{7}$. Su versión en español, estandarizada en población chilena, además correlacionó estos resultados con el índice de Karnofsky y el cuestionario sobre la salud del paciente-9 (GHQ-9) utilizado para la pesquisa de depresión ${ }^{8}$. Los resultados emanados de nuestros pacientes se asemejan con los obtenidos por Nazar et al., quienes evidencian, además, la relación entre nivel educacional y el grado de asistencia requerido para la respuesta del cuestionario ${ }^{8}$, lo cual se pudo observar en nuestros pacientes. En el presente estudio, la subescala de función física, que incluye los dominios de deglución y masticación, registró un puntaje promedio más alto que el obtenido en la subescala socioemocional, lo que refleja la importancia de identificar y manejar de manera multidisciplinaria factores biopsicosociales que impactan negativamente en la calidad de vida postratamiento.

\section{Conclusiones}

La reconstrucción faríngea parcial con colgajo miocutáneo pediculado en pacientes tratados con laringectomías totales asociadas a faringectomía parcial permite mantener una deglución adecuada y sin disfagia, con un estado nutricional óptimo y sin evidenciar impacto negativo significativo en la calidad de vida del paciente. Recomendamos su uso como alternativa a los colgajos libres en pacientes seleccionados.

\section{Responsabilidades éticas}

Protección de personas y animales. Los autores declaran que para esta investigación no se han realizado experimentos en seres humanos ni en animales.

Confidencialidad de los datos. Los autores declaran que en este artículo no aparecen datos de pacientes.

Conflictos de interés: no hay.

\section{Referencias}

1. Siegel RL, Miller KD, Jemal A. Cancer Statistics 2017. CA Cancer J Clin. 2017;67:7-30.

2. Ortega FG, Cardemil MF. Estimación de la incidencia del cáncer de laringe en Chile según la aplicación de un formulario de registro digital. Revista de otorrinolaringología y cirugía de cabeza y cuello 2017;77:150-6.

3. Marur S, Forastiere AA. Head and Neck Squamous Cell Carcinoma: Update on Epidemiology, Diagnosis and Treatment. Mayo Clin Proc. 2016;91:386-96.

4. Steuer CE, El-Deiry M, Parks JR, Higgins KA, Saba NF. An update on larynx cancer. CA Cancer J Clin. 2017;67:31-50.

5. Van der Putten L, Spasiano R, de Bree R, Bertino G, Leemans CR, Benazzo M. Flap reconstruction of the hypopharynx: a defect orientated approach. Acta Otorhinolaryngol Ital. 2012;32:288-96.

6. Chao JW, Spector JA, Taylor EM, Otterburn DM, Kutler DI, Caruana SM, et al. Pectoralis major myocutaneous flap versus free fasciocutaneous flap for reconstruction of partial hypopharyngeal defects: what should we be doing?

J Reconstr Microsurg. 2015;31:198-204.

7. Lowe DRS. University of Washington Quality of Life Questionnaire (UW-QOL v4). 2012:1-11.

8. Nazar G, Garmendia ML, Royer M, McDowell JA, Weymuller EA Jr, Yueh B. Spanish validation of the University of Washington Quality of Life questionnaire for head and neck cancer patients. Otolaryngol Head Neck Surg. 2010;143:801-7, e801-2.

9. Genden EM, Ferlito A, Silver CE, Jacobson AS, Werner JA, Suárez C, et al. Evolution of the management of laryngeal cancer. Oral Oncology 2007;43:431-9.

10. Yáñez MR, Loyola BFJ, Urzúa EC, Cornejo FJ. Laringectomía total ampliada en carcinoma laríngeo avanzado T4a. Rev Chil Cir. 2012;64:442-6.

11. Pradenas MVM, Rahal M, Cisternas A. Cáncer de laringe e hipofaringe. Experiencia del Hospital Barros Luco Trudeau 1990-2001. Revista de otorrinolaringología y cirugía de cabeza y cuello. 2004;64:13-20.

12. Welkoborsky HJ, Deichmüller C, Bauer L, Hinni ML. Reconstruction of large pharyngeal defects with microvascular free flaps and myocutaneous pedicled flaps. Curr Opin Otolaryngol Head Neck Surg. 2013;21:318-27.

13. Virós Porcuna D, León Vintró X, López Vilas M, Pujol Olmo A, Masià Ayala J, Quer Agustí M. Pectoralis Major Flaps. Evolution of Their Use in the Age of Microvascularized Flaps. Acta Otorrinolaringológica (English Edition). 2008;59:263-8.

14. Clark JR, Gilbert R, Irish J, Brown D, Neligan P, Gullane PJ. Morbidity after flap reconstruction of hypopharyngeal defects. Laryngoscope 2006; 116 : 173-81.

15. Wong $\mathrm{CH}$, Wei FC. Microsurgical free flap in head and neck reconstruction. Head Neck 2010;32:1236-45.

16. Lee T, Chung C, Chang Y, Kim J. Comparison of Clinical and Functional Outcomes Using Pectoralis Major and Cutaneous Free Flaps for Hypopharyngeal Squamous Cell Carcinoma. Arch Plast Surg. 2015;42:608-13.

17. Hamlet SL, Wilson S, Stachler RJ, Salwen WA, Muz J, Heilbrun LK. Scintigraphic assessment of swallow efficiency postlaryngectomy. The Laryngoscope. 1994;104:1159-62. 
18. Dos Santos Queija D, Portas JG, Dedivitis RA, Lehn CN, Barros APB. Swallowing and quality of life after total laryngectomy and pharyngolaryngectomy. Brazilian Journal of Otorhinolaryngology 2009; 75:556-64.

19. Marks JE, Freeman RB, Lee F, Ogura JH. Pharyngeal wall cancer: an analysis of treatment results complications and patterns of failure. Int J Radiat Oncol Biol Phys. 1978;4:587-93.
20. Haubner F, Ohmann E, Pohl F, Strutz J, Gassner HG. Wound healing after radiation therapy: Review of the literature. Radiation Oncology. 2012;7:162. doi: 10.1186/1748-717X-7-162

21. Morgan JE, Breau RL, Suen JY, Hanna EY. Surgical wound complications after intensive chemoradiotherapy for advanced squamous cell carcinoma of the head and neck. Archives of Otolaryngology-Head \& Neck Surgery. 2007;133:10-4.
22. Bhattacharjee A, Bahar I, Saikia A. Nutritional Assessment of Patients with Head and Neck Cancer in North-East India and Dietary Intervention. Indian J Palliat Care 2015;21:289-95.

23. Prevost V, Joubert C, Heutte N, Babin E. Assessment of nutritional status and quality of life in patients treated for head and neck cancer. European Annals of Otorhinolaryngology, Head and Neck Diseases 2014;131:113-20. 